\title{
New existence and uniqueness results for an elastic beam equation with nonlinear boundary conditions
}

\author{
Shunyong $\mathrm{Li}^{1,2}$ and Chengbo Zhai ${ }^{*}$
}

"Correspondence:
cbzhai@sxu.edu.cn
'School of Mathematical Sciences,
Shanxi University, Taiyuan, Shanxi
030006, P.R. China
Full list of author information is
available at the end of the article

\section{Springer}

\begin{abstract}
In this article, we study the existence and uniqueness of monotone positive solutions for an elastic beam equation with nonlinear boundary conditions, and some sufficient conditions which guarantee the existence of unique monotone positive solution are established. The methods employed are two fixed point theorems for mixed monotone operators with perturbation. Our results can not only guarantee the existence of unique monotone positive solution, but also be applied to construct an iterative scheme for approximating it. Two examples are given to illustrate our main results.
\end{abstract}

MSC: 34B18;34B15

Keywords: existence and uniqueness; monotone positive solution; elastic beam equation; fixed point theorem for mixed monotone operators

\section{Introduction}

In this article, we are concerned with the existence and uniqueness of monotone positive solutions for the following nonlinear fourth-order two-point boundary value problem for elastic beam equation:

$$
\left\{\begin{array}{l}
u^{(4)}(t)=f\left(t, u(t), u^{\prime}(t)\right), \quad 0<t<1, \\
u(0)=u^{\prime}(0)=0, \\
u^{\prime \prime}(1)=0, \quad u^{(3)}(1)=g(u(1)),
\end{array}\right.
$$

where $f \in C([0,1] \times \mathbf{R} \times \mathbf{R})$ and $g \in C(\mathbf{R})$ are real functions. Here, monotone positive solutions mean increasing positive solutions. As we know, problem (1.1) models an elastic beam of length 1 subject to a nonlinear foundation given by the function $f$. The first boundary condition $u(0)=u^{\prime}(0)=0$ means that the left end of the beam is fixed. The second boundary condition $u^{\prime \prime}(1)=0, u^{\prime \prime \prime}(1)=g(u(1))$ means that the right end of the beam is attached to a bearing device, given by the function $g$. Owing to the importance in engineering, physics and material mechanics, boundary value problems for elastic beam equations have attracted much attention. For a small sample of such work, we refer the reader to the works [1-20] and the references therein. In these papers, most of the authors have investigated the existence of solutions or positive solutions. On the other hand, the unique-

(c) $2015 \mathrm{Li}$ and Zhai. This article is distributed under the terms of the Creative Commons Attribution 4.0 International License (http://creativecommons.org/licenses/by/4.0/), which permits unrestricted use, distribution, and reproduction in any medium, provided you give appropriate credit to the original author(s) and the source, provide a link to the Creative Commons license, and indicate if changes were made. 
ness of positive solutions for nonlinear fourth-order boundary value problems has been studied by some authors, see [16, 17, 19] for example. In [17], the authors utilized a fixed point theorem of generalized concave operators to study problem (1.1) and established the existence and uniqueness of monotone positive solutions. In [19], by using a fixed point theorem of cone expansion and a fixed point theorem of generalized concave operators, the authors considered the existence, nonexistence, and uniqueness of convex monotone positive solutions of an elastic beam equation with a parameter.

Motivated by the work [17, 21], we will discuss the existence and uniqueness of monotone positive solutions for problem (1.1) by using two fixed point theorems for mixed monotone operators with perturbation. As we know, there are still very few works to utilize fixed point theorems of mixed monotone operators to study fourth-order boundary value problems. So it is worthwhile to investigate problem (1.1) and the methods used here are relatively new to the literature. The main features of this article are as follows. First, we consider the monotone positive solutions for fourth-order boundary value problems. Second, comparing with $[16,17,19]$, we establish the existence and uniqueness of monotone positive solutions via different methods. Third, our results can not only guarantee the existence of a unique monotone positive solution, but also be applied to construct an iterative scheme for approximating it. In addition, few papers can be found in the literature on the existence and uniqueness of monotone positive solutions for fourth-order boundary value problems. Hence we improve the results of [17] to some degree, and so it is important to study the existence and uniqueness of monotone positive solutions for problem (1.1).

\section{Preliminaries}

In the following, for completeness we list some basic concepts in ordered Banach spaces and two fixed point theorems for mixed monotone operators which will be used later. For the convenience of readers, we refer them to [21-23] for details.

Let $(E,\|\cdot\|)$ be a real Banach space which is partially ordered by a cone $P \subset E$, i.e., $x \leq y$ if and only if $y-x \in P$. If $x \leq y$ and $x \neq y$, then we denote $x<y$ or $y>x$. By $\theta$ we denote the zero element of $E$. A non-empty closed convex set $P \subset E$ is a cone if it satisfies (i) $x \in P$, $\lambda \geq 0 \Rightarrow \lambda x \in P$; (ii) $x \in P,-x \in P \Rightarrow x=\theta$.

$P$ is called normal if there is a constant $N>0$ such that, for all $x, y \in E, \theta \leq x \leq y$ implies $\|x\| \leq N\|y\|$; in this case $N$ is the infimum of such constants, it is called the normality constant of $P$. If $x_{1}, x_{2} \in E$, the set $\left[x_{1}, x_{2}\right]=\left\{x \in E \mid x_{1} \leq x \leq x_{2}\right\}$ is called the order interval between $x_{1}$ and $x_{2}$. We say that an operator $A: E \rightarrow E$ is increasing (decreasing) if $x \leq y$ implies $A x \leq A y(A x \geq A y)$.

For all $x, y \in E$, the notation $x \sim y$ means that there exist $\lambda>0$ and $\mu>0$ such that $\lambda x \leq y \leq \mu x$. Clearly, $\sim$ is an equivalence relation. Given $h>\theta$ (i.e., $h \geq \theta$ and $h \neq \theta$ ), we denote by $P_{h}$ the set $P_{h}=\{x \in E \mid x \sim h\}$. It is easy to see that $P_{h} \subset P$.

Definition 2.1 (See [21, 23]) $A: P \times P \rightarrow P$ is said to be a mixed monotone operator if $A(x, y)$ is increasing in $x$ and decreasing in $y$, i.e., $u_{i}, v_{i} \in P, i=1,2, u_{1} \leq u_{2}, v_{1} \geq v_{2}$ imply $A\left(u_{1}, v_{1}\right) \leq A\left(u_{2}, v_{2}\right)$. Element $x \in P$ is called a fixed point of $A$ if $A(x, x)=x$.

Definition 2.2 An operator $A: P \rightarrow P$ is said to be sub-homogeneous if it satisfies

$$
A(t x) \geq t A(x), \quad \forall t \in(0,1), x \in P .
$$


Definition 2.3 Let $\alpha$ be a real number with $0 \leq \alpha<1$. An operator $A: P \rightarrow P$ is said to be $\alpha$-concave if it satisfies

$$
A(t x) \geq t^{\alpha} A(x), \quad \forall t \in(0,1), x \in P .
$$

To prove our results, we need the following fixed point theorems for mixed monotone operators, which were established in [21].

Lemma 2.4 (See Theorem 2.1 in [21]) Let $h>\theta$ and $\alpha \in(0,1) . A: P \times P \rightarrow P$ is a mixed monotone operator and satisfies

$$
A\left(t x, t^{-1} y\right) \geq t^{\alpha} A(x, y), \quad \forall t \in(0,1), x, y \in P .
$$

$B: P \rightarrow P$ is an increasing sub-homogeneous operator. Suppose that

(i) there is $h_{0} \in P_{h}$ such that $A\left(h_{0}, h_{0}\right) \in P_{h}$ and $B h_{0} \in P_{h}$;

(ii) there exists a constant $\delta_{0}>0$ such that $A(x, y) \geq \delta_{0} B x, \forall x, y \in P$.

Then:

(1) $A: P_{h} \times P_{h} \rightarrow P_{h}$ and $B: P_{h} \rightarrow P_{h}$;

(2) there exist $u_{0}, v_{0} \in P_{h}$ and $r \in(0,1)$ such that

$$
r v_{0} \leq u_{0}<v_{0}, \quad u_{0} \leq A\left(u_{0}, v_{0}\right)+B u_{0} \leq A\left(v_{0}, u_{0}\right)+B v_{0} \leq v_{0}
$$

(3) the operator equation $A(x, x)+B x=x$ has a unique solution $x^{*}$ in $P_{h}$;

(4) for any initial values $x_{0}, y_{0} \in P_{h}$, constructing successively the sequences

$$
x_{n}=A\left(x_{n-1}, y_{n-1}\right)+B x_{n-1}, \quad y_{n}=A\left(y_{n-1}, x_{n-1}\right)+B y_{n-1}, \quad n=1,2, \ldots,
$$

we have $x_{n} \rightarrow x^{*}$ and $y_{n} \rightarrow x^{*}$ as $n \rightarrow \infty$.

Lemma 2.5 (See Theorem 2.4 in [21]) Let $h>\theta$ and $\alpha \in(0,1) . A: P \times P \rightarrow P$ is a mixed monotone operator and satisfies

$$
A\left(t x, t^{-1} y\right) \geq t A(x, y), \quad \forall t \in(0,1), x, y \in P .
$$

$B: P \rightarrow P$ is an increasing $\alpha$-concave operator. Suppose that

(i) there is $h_{0} \in P_{h}$ such that $A\left(h_{0}, h_{0}\right) \in P_{h}$ and $B h_{0} \in P_{h}$;

(ii) there exists a constant $\delta_{0}>0$ such that $A(x, y) \leq \delta_{0} B x, \forall x, y \in P$.

Then:

(1) $A: P_{h} \times P_{h} \rightarrow P_{h}$ and $B: P_{h} \rightarrow P_{h}$;

(2) there exist $u_{0}, v_{0} \in P_{h}$ and $r \in(0,1)$ such that

$$
r v_{0} \leq u_{0}<v_{0}, \quad u_{0} \leq A\left(u_{0}, v_{0}\right)+B u_{0} \leq A\left(v_{0}, u_{0}\right)+B v_{0} \leq v_{0} ;
$$

(3) the operator equation $A(x, x)+B x=x$ has a unique solution $x^{*}$ in $P_{h}$;

(4) for any initial values $x_{0}, y_{0} \in P_{h}$, constructing successively the sequences

$$
x_{n}=A\left(x_{n-1}, y_{n-1}\right)+B x_{n-1}, \quad y_{n}=A\left(y_{n-1}, x_{n-1}\right)+B y_{n-1}, \quad n=1,2, \ldots,
$$

we have $x_{n} \rightarrow x^{*}$ and $y_{n} \rightarrow x^{*}$ as $n \rightarrow \infty$. 


\section{Main results}

In this section, we use Lemmas 2.4, 2.5 to study problem (1.1) and present two new results on the existence and uniqueness of monotone positive solutions. The main results obtained here are relatively new in the literature.

In our considerations we shall consider the Banach space $E=C^{1}[0,1]$ equipped with the norm $\|u\|=\max \left\{\max _{0 \leq t \leq 1}|u(t)|, \max _{0 \leq t \leq 1}\left|u^{\prime}(t)\right|\right\}$. In order to find monotone positive solutions, we consider the closed convex cone of nonnegative increasing functions $P=$ $\left\{u \in E \mid u(t) \geq 0, u^{\prime}(t) \geq 0, \forall t \in[0,1]\right\}$. Note that this induces an order relation $\dot{\leq}$ in $E$ by defining $u \dot{\leq} v$ if and only if $v-u \in P$. Clearly, this cone is normal. That is, if $u \dot{\leq} v$, then $u(t) \leq v(t), u^{\prime}(t) \leq v^{\prime}(t), t \in[0,1]$. Therefore, $\|u\| \leq\|v\|$ and the normality constant is 1.

Let $G(t, s)$ be the Green function of the linear problem $u^{(4)}(t)=0$ with the boundary conditions in (1.1); from [3] we know that

$$
G(t, s)=\frac{1}{6}\left\{\begin{array}{cc}
s^{2}(3 t-s), & 0 \leq s \leq t \leq 1 \\
t^{2}(3 s-t), & 0 \leq t \leq s \leq 1
\end{array}\right.
$$

Then problem (1.1) is equivalent to the integral equation

$$
u(t)=\int_{0}^{1} G(t, s) f\left(s, u(s), u^{\prime}(s)\right) d s-g(u(1)) \phi(t), \quad \text { where } \phi(t)=\frac{1}{2} t^{2}-\frac{1}{6} t^{3}, t \in[0,1] .
$$

From [17], we give the following properties of the Green function $G(t, s)$ and $\phi(t)$.

Lemma 3.1 For any $t, s \in[0,1]$, we have

$$
\begin{aligned}
& \frac{1}{3} s^{2} t^{2} \leq G(t, s) \leq \frac{1}{2} s t^{2}, \quad \frac{1}{3} t^{2} \leq \phi(t) \leq \frac{1}{2} t^{2}, \\
& \frac{1}{2} s^{2} t \leq \frac{\partial G(t, s)}{\partial t} \leq s t, \quad \frac{1}{2} t \leq \phi^{\prime}(t) \leq 2 t .
\end{aligned}
$$

\section{Theorem 3.2 Assume that}

$\left(\mathrm{H}_{1}\right) f(t, x, y):[0,1] \times[0,+\infty) \times[0,+\infty) \rightarrow[0,+\infty)$ and $g:[0,+\infty) \rightarrow(-\infty, 0] ;$

$\left(\mathrm{H}_{2}\right) f(t, x, y)$ is increasing in $x \in[0,+\infty)$ for fixed $t \in[0,1]$ and $y \in[0,+\infty)$, decreasing in $y \in[0,+\infty)$ for fixed $t \in[0,1]$ and $x \in[0,+\infty)$, and $g(x)$ is decreasing in $x \in[0,+\infty)$;

$\left(\mathrm{H}_{3}\right) g(\lambda x) \leq \lambda g(x)$ for $\lambda \in(0,1), x \in[0,+\infty)$, and there exists a constant $\alpha \in(0,1)$ such that $f\left(t, \lambda x, \lambda^{-1} y\right) \geq \lambda^{\alpha} f(t, x, y), \forall t \in[0,1], \lambda \in(0,1), x, y \in[0,+\infty) ;$

$\left(\mathrm{H}_{4}\right)$ there exists a constant $\sigma>0$ such that $f(t, x, y) \geq \sigma \geq-g(x)>0, t \in[0,1], x, y \geq 0$.

Then:

(1) there exist $u_{0}, v_{0} \in P_{h}$ and $r \in(0,1)$ such that $r v_{0} \dot{\leq} u_{0} \dot{<} v_{0}$ and

$$
\begin{aligned}
& u_{0}(t) \leq \int_{0}^{1} G(t, s) f\left(s, u_{0}(s), v_{0}^{\prime}(s)\right) d s-g\left(u_{0}(1)\right) \phi(t), \quad t \in[0,1], \\
& u_{0}^{\prime}(t) \leq \int_{0}^{1} G_{t}(t, s) f\left(s, u_{0}(s), v_{0}^{\prime}(s)\right) d s-g\left(u_{0}(1)\right) \phi^{\prime}(t), \quad t \in[0,1], \\
& v_{0}(t) \geq \int_{0}^{1} G(t, s) f\left(s, v_{0}(s), u_{0}^{\prime}(s)\right) d s-g\left(v_{0}(1)\right) \phi(t), \quad t \in[0,1],
\end{aligned}
$$




$$
v_{0}^{\prime}(t) \geq \int_{0}^{1} G_{t}(t, s) f\left(s, v_{0}(s), u_{0}^{\prime}(s)\right) d s-g\left(v_{0}(1)\right) \phi^{\prime}(t), \quad t \in[0,1]
$$

where $h(t)=t^{2}, t \in[0,1]$ and $G(t, s)$ is given as in (3.1);

(2) problem (1.1) has a unique monotone positive solution $u^{*}$ in $P_{h}$;

(3) for any $x_{0}, y_{0} \in P_{h}$, constructing successively the sequences

$$
\begin{aligned}
& x_{n}(t)=\int_{0}^{1} G(t, s) f\left(s, x_{n-1}(s), y_{n-1}^{\prime}(s)\right) d s-g\left(x_{n-1}(1)\right) \phi(t), \quad n=1,2, \ldots, \\
& y_{n}(t)=\int_{0}^{1} G(t, s) f\left(s, y_{n-1}(s), x_{n-1}^{\prime}(s)\right) d s-g\left(y_{n-1}(1)\right) \phi^{\prime}(t), \quad n=1,2, \ldots
\end{aligned}
$$

we have $\left\|x_{n}-u^{*}\right\| \rightarrow 0$ and $\left\|y_{n}-u^{*}\right\| \rightarrow 0$ as $n \rightarrow \infty$.

Proof Define two operators $A: P \times P \rightarrow E$ and $B: P \rightarrow E$ by

$$
A(u, v)(t)=\int_{0}^{1} G(t, s) f\left(s, u(s), v^{\prime}(s)\right) d s, \quad B u(t)=-g(u(1)) \phi(t), \quad t \in[0,1] .
$$

Then

$$
(A(u, v))^{\prime}(t)=\int_{0}^{1} G_{t}(t, s) f\left(s, u(s), v^{\prime}(s)\right) d s, \quad(B u)^{\prime}(t)=-g(u(1)) \phi^{\prime}(t), \quad t \in[0,1] .
$$

Evidently, $u$ is the solution of problem (1.1) if and only if $u=A(u, u)+B u$. For $u, v \in P$, we know that $u(t), v(t) \geq 0, u^{\prime}(t), v^{\prime}(t) \geq 0, t \in[0,1]$. From $\left(\mathrm{H}_{1}\right)$ and Lemma 3.1, we have $A(u, v)(t) \geq 0, B u(t) \geq 0,(A(u, v))^{\prime}(t) \geq 0,(B u)^{\prime}(t) \geq 0, t \in[0,1]$. Therefore, $A(u, v) \in P$, $B u \in P$. That is, $A: P \times P \rightarrow P$ and $B: P \rightarrow P$. In the sequel we check that $A, B$ satisfy all assumptions of Lemma 2.4.

Firstly, we prove that $A$ is a mixed monotone operator. In fact, for $u_{i}, v_{i} \in P, i=1,2$ with $u_{1} \dot{\geq} u_{2}, v_{1} \dot{\leq} v_{2}$, we know that $u_{1}(t) \geq u_{2}(t), v_{1}(t) \leq v_{2}(t), u_{1}^{\prime}(t) \geq u_{2}^{\prime}(t), v_{1}^{\prime}(t) \leq v_{2}^{\prime}(t), t \in[0,1]$ and by $\left(\mathrm{H}_{2}\right)$ and Lemma 3.1, we have

$$
\begin{aligned}
A\left(u_{1}, v_{1}\right)(t) & =\int_{0}^{1} G(t, s) f\left(s, u_{1}(s), v_{1}^{\prime}(s)\right) d s \\
& \geq \int_{0}^{1} G(t, s) f\left(s, u_{2}(s), v_{2}^{\prime}(s)\right) d s \\
& =A\left(u_{2}, v_{2}\right)(t), \\
\left(A\left(u_{1}, v_{1}\right)\right)^{\prime}(t) & =\int_{0}^{1} G_{t}(t, s) f\left(s, u_{1}(s), v_{1}^{\prime}(s)\right) d s \\
& \geq \int_{0}^{1} G_{t}(t, s) f\left(s, u_{2}(s), v_{2}^{\prime}(s)\right) d s \\
& =\left(A\left(u_{2}, v_{2}\right)\right)^{\prime}(t) .
\end{aligned}
$$

That is, $A\left(u_{1}, v_{1}\right) \dot{\geq} A\left(u_{2}, v_{2}\right)$.

Further, we show $B$ is increasing. For any $u, v \in P$ with $u \leq v$, we know that $u(t) \leq v(t)$, $u^{\prime}(t) \leq v^{\prime}(t), t \in[0,1]$. It follows from $\left(\mathrm{H}_{1}\right),\left(\mathrm{H}_{2}\right)$ and Lemma 3.1 that $B u(t) \leq B v(t)$, 
$(B u)^{\prime}(t) \leq(B v)^{\prime}(t), t \in[0,1]$. That is, $B u \dot{\leq} B v$. Next we show that operator $A$ satisfies the condition (2.1). For any $\lambda \in(0,1)$ and $u, v \in P$, by $\left(\mathrm{H}_{3}\right)$ we have

$$
\begin{aligned}
A\left(\lambda u, \lambda^{-1} v\right)(t) & =\int_{0}^{1} G(t, s) f\left(s, \lambda u(s), \lambda^{-1} v^{\prime}(s)\right) d s \\
& \geq \lambda^{\alpha} \int_{0}^{1} G(t, s) f\left(s, u(s), v^{\prime}(s)\right) d s \\
& =\lambda^{\alpha} A(u, v)(t), \\
\left(A\left(\lambda u, \lambda^{-1} v\right)\right)^{\prime}(t) & =\int_{0}^{1} G_{t}(t, s) f\left(s, \lambda u(s), \lambda^{-1} v^{\prime}(s)\right) d s \\
& \geq \lambda^{\alpha} \int_{0}^{1} G(t, s) f\left(s, u(s), v^{\prime}(s)\right) d s \\
& =\left(\lambda^{\alpha} A(u, v)\right)^{\prime}(t) .
\end{aligned}
$$

That is, $A\left(\lambda u, \lambda^{-1} v\right) \dot{\geq} \lambda^{\alpha} A(u, v)$ for $\lambda \in(0,1), u, v \in P$. So operator $A$ satisfies (2.1). Also, for any $\lambda \in(0,1), u \in P$, from $\left(\mathrm{H}_{3}\right)$ we know that

$$
\begin{aligned}
& B(\lambda u)(t)=-g(\lambda u(1)) \phi(t) \geq \lambda(-g(u(1)) \phi(t))=\lambda B u(t), \\
& (B(\lambda u))^{\prime}(t)=-g(\lambda u(1)) \phi^{\prime}(t) \geq \lambda\left(-g(u(1)) \phi^{\prime}(t)\right)=(\lambda B u)^{\prime}(t),
\end{aligned}
$$

that is, $B(\lambda u) \geq \lambda B u$ for $\lambda \in(0,1), u \in P$. That is, operator $B$ is sub-homogeneous. Now we show that $A(h, h) \in P_{h}$ and $B h \in P_{h}$. On the one hand, from $\left(\mathrm{H}_{1}\right),\left(\mathrm{H}_{2}\right)$ and Lemma 3.1, for any $t \in[0,1]$, we have

$$
\begin{aligned}
A(h, h)(t) & =\int_{0}^{1} G(t, s) f\left(s, h(s), h^{\prime}(s)\right) d s \\
& =\int_{0}^{1} G(t, s) f\left(s, s^{2}, 2 s\right) d s \\
& \leq \int_{0}^{1} \frac{1}{2} t^{2} s f\left(s, s^{2}, 2 s\right) d s \leq \frac{1}{2} \int_{0}^{1} s f(s, 1,0) d s \cdot h(t), \\
A(h, h)(t) & \geq \int_{0}^{1} \frac{1}{3} t^{2} s^{2} f\left(s, s^{2}, 2 s\right) d s \geq \frac{1}{3} \int_{0}^{1} s^{2} f(s, 0,2) d s \cdot h(t) .
\end{aligned}
$$

On the other hand, also from $\left(\mathrm{H}_{1}\right),\left(\mathrm{H}_{2}\right)$ and Lemma 3.1, for any $t \in[0,1]$, we obtain

$$
\begin{aligned}
(A(h, h))^{\prime}(t) & =\int_{0}^{1} G_{t}(t, s) f\left(s, s^{2}, 2 s\right) d s \\
& \leq \int_{0}^{1} s t f(s, 1,0) d s=\frac{1}{2} \int_{0}^{1} s f(s, 1,0) d s \cdot h^{\prime}(t), \\
(A(h, h))^{\prime}(t) & \geq \int_{0}^{1} \frac{1}{2} s^{2} t f(s, 0,2) d s=\frac{1}{4} \int_{0}^{1} s^{2} f(s, 0,2) d s \cdot h^{\prime}(t) .
\end{aligned}
$$

Let

$$
c_{1}=\frac{1}{4} \int_{0}^{1} s^{2} f(s, 0,2) d s, \quad c_{2}=\frac{1}{2} \int_{0}^{1} s f(s, 1,0) d s .
$$


From $\left(\mathrm{H}_{2}\right),\left(\mathrm{H}_{4}\right)$, we have

$$
c_{2} \geq c_{1} \geq \frac{1}{4} \int_{0}^{1} s^{2} \sigma d s=\frac{1}{12} \sigma>0,
$$

and in consequence,

$$
\begin{aligned}
& c_{1} h(t) \leq A(h, h)(t) \leq c_{2} h(t), \\
& \left(c_{1} h\right)^{\prime}(t)=c_{1} h^{\prime}(t) \leq(A(h, h))^{\prime}(t) \leq c_{2} h^{\prime}(t)=\left(c_{2} h\right)^{\prime}(t), \quad t \in[0,1] .
\end{aligned}
$$

Thus, $c_{1} h \dot{\leq} A(h, h) \dot{\leq} c_{2} h$. That is, $A(h, h) \in P_{h}$. Similarly, from $\left(\mathrm{H}_{1}\right),\left(\mathrm{H}_{2}\right)$ and Lemma 3.1, for any $t \in[0,1]$, we have

$$
\begin{aligned}
& -\frac{1}{3} g(1) h(t)=-g(1) \frac{1}{3} t^{2} \leq B h(t)=-g(h(1)) \phi(t) \leq-g(1) \frac{1}{2} t^{2}=-\frac{1}{2} g(1) h(t), \\
& -\frac{1}{4} g(1) h^{\prime}(t)=-g(1) \frac{1}{2} t \leq(B h)^{\prime}(t)=-g(h(1)) \phi^{\prime}(t) \leq-g(1) 2 t=-g(1) h^{\prime}(t) .
\end{aligned}
$$

Let $c_{3}=-\frac{1}{4} g(1), c_{4}=-g(1)$. Then, from $\left(\mathrm{H}_{1}\right),\left(\mathrm{H}_{4}\right)$, we have $c_{4} \geq c_{3}>0$ and thus

$$
\begin{aligned}
& c_{3} h(t) \leq B h(t) \leq c_{4} h(t), \\
& \left(c_{3} h\right)^{\prime}(t)=c_{3} h^{\prime}(t) \leq(B h)^{\prime}(t) \leq c_{4} h^{\prime}(t)=\left(c_{4} h\right)^{\prime}(t), \quad t \in[0,1] .
\end{aligned}
$$

Therefore, $c_{3} h \dot{\leq} B h \dot{\leq} c_{4} h$. That is, $B h \in P_{h}$. Hence the condition (i) of Lemma 2.4 is satisfied.

In the following we show the condition (ii) of Lemma 2.4 is satisfied. For $u, v \in P$ and any $t \in[0,1]$, from $\left(\mathrm{H}_{4}\right)$ and Lemma 3.1,

$$
\begin{aligned}
A(u, v)(t) & =\int_{0}^{1} G(t, s) f\left(s, u(s), v^{\prime}(s)\right) d s \\
& \geq \int_{0}^{1} \frac{1}{3} t^{2} s^{2} f\left(s, u(s), v^{\prime}(s)\right) d s \geq \frac{1}{3} t^{2} \int_{0}^{1} s^{2} \sigma d s \\
& =\frac{1}{9} \sigma t^{2} \geq \frac{1}{9} t^{2}[-g(u(1))]=\frac{2}{9}[-g(u(1))] \frac{1}{2} t^{2} \\
\geq & \frac{2}{9}[-g(u(1))] \phi(t)=\frac{2}{9} B u(t), \\
(A(u, v))^{\prime}(t) & =\int_{0}^{1} G(t, s) f\left(s, u(s), v^{\prime}(s)\right) d s \\
& \geq \int_{0}^{1} \frac{1}{2} t s^{2} f\left(s, u(s), v^{\prime}(s)\right) d s \geq \frac{1}{2} t \int_{0}^{1} s^{2} \sigma d s \\
& =\frac{1}{6} \sigma t \geq \frac{1}{6} t[-g(u(1))]=\frac{1}{12}[-g(u(1))] 2 t \\
& \geq \frac{1}{12}[-g(u(1))] \phi^{\prime}(t)=\frac{1}{12}(B u)^{\prime}(t) .
\end{aligned}
$$

Let $\delta_{0}=\frac{1}{12}$. Then

$$
A(u, v)(t) \geq \delta_{0} B u(t), \quad(A(u, v))^{\prime}(t) \geq \delta_{0}(B u)^{\prime}(t), \quad t \in[0,1] .
$$


Therefore, we get $A(u, v) \dot{\geq} \delta_{0} B u$ for $u, v \in P$. Finally, an application of Lemma 2.4 implies: there exist $u_{0}, v_{0} \in P_{h}$ and $r \in(0,1)$ such that $r v_{0} \dot{\leq} u_{0} \dot{<} v_{0}, u_{0} \dot{\leq} A\left(u_{0}, v_{0}\right)+B u_{0} \dot{\leq} A\left(v_{0}, u_{0}\right)+$ $B v_{0} \dot{\leq} v_{0}$; the operator equation $A(u, u)+B u=u$ has a unique solution $u^{*}$ in $P_{h}$; for any initial values $x_{0}, y_{0} \in P_{h}$, constructing successively the sequences

$$
x_{n}=A\left(x_{n-1}, y_{n-1}\right)+B x_{n-1}, \quad y_{n}=A\left(y_{n-1}, x_{n-1}\right)+B y_{n-1}, \quad n=1,2, \ldots,
$$

we have $x_{n} \rightarrow u^{*}$ and $y_{n} \rightarrow u^{*}$ as $n \rightarrow \infty$. That is,

$$
\begin{aligned}
& u_{0}(t) \leq \int_{0}^{1} G(t, s) f\left(s, u_{0}(s), v_{0}^{\prime}(s)\right) d s-g\left(u_{0}(1)\right) \phi(t), \quad t \in[0,1], \\
& u_{0}^{\prime}(t) \leq \int_{0}^{1} G_{t}(t, s) f\left(s, u_{0}(s), v_{0}^{\prime}(s)\right) d s-g\left(u_{0}(1)\right) \phi^{\prime}(t), \quad t \in[0,1], \\
& v_{0}(t) \geq \int_{0}^{1} G(t, s) f\left(s, v_{0}(s), u_{0}^{\prime}(s)\right) d s-g\left(v_{0}(1)\right) \phi(t), \quad t \in[0,1], \\
& v_{0}^{\prime}(t) \geq \int_{0}^{1} G_{t}(t, s) f\left(s, v_{0}(s), u_{0}^{\prime}(s)\right) d s-g\left(v_{0}(1)\right) \phi^{\prime}(t), \quad t \in[0,1]
\end{aligned}
$$

problem (1.1) has a unique positive solution $u^{*}$ in $P_{h}$ and $u^{*}(t)$ is monotone increasing; for any $x_{0}, y_{0} \in P_{h}$, constructing successively the sequences

$$
\begin{aligned}
& x_{n}(t)=\int_{0}^{1} G(t, s) f\left(s, x_{n-1}(s), y_{n-1}^{\prime}(s)\right) d s-g\left(x_{n-1}(1)\right) \phi(t), \quad n=1,2, \ldots, \\
& y_{n}(t)=\int_{0}^{1} G(t, s) f\left(s, y_{n-1}(s), x_{n-1}^{\prime}(s)\right) d s-g\left(y_{n-1}(1)\right) \phi^{\prime}(t), \quad n=1,2, \ldots
\end{aligned}
$$

we have $\left\|x_{n}-u^{*}\right\| \rightarrow 0$ and $\left\|y_{n}-u^{*}\right\| \rightarrow 0$ as $n \rightarrow \infty$.

Theorem 3.3 Assume $\left(\mathrm{H}_{1}\right),\left(\mathrm{H}_{2}\right)$ and

$\left(\mathrm{H}_{5}\right)$ there exists a constant $\alpha \in(0,1)$ such that $g(\lambda x) \leq \lambda^{\alpha} g(x), \forall \lambda \in(0,1), x \in[0,+\infty)$, and $f\left(t, \lambda x, \lambda^{-1} y\right) \geq \lambda f(t, x, y)$ for $\lambda \in(0,1), t \in[0,1], x, y \in[0,+\infty) ;$

$\left(\mathrm{H}_{6}\right) f(t, 0,2) \not \equiv 0$ for $t \in[0,1]$ and there exists a constant $\sigma>0$ such that $f(t, x, y) \leq \sigma \leq$ $-g(x), t \in[0,1], x, y \geq 0$.

Then:

(1) there exist $u_{0}, v_{0} \in P_{h}$ and $r \in(0,1)$ such that $r v_{0} \dot{\leq} u_{0} \dot{<} v_{0}$ and

$$
\begin{aligned}
& u_{0}(t) \leq \int_{0}^{1} G(t, s) f\left(s, u_{0}(s), v_{0}^{\prime}(s)\right) d s-g\left(u_{0}(1)\right) \phi(t), \quad t \in[0,1], \\
& u_{0}^{\prime}(t) \leq \int_{0}^{1} G_{t}(t, s) f\left(s, u_{0}(s), v_{0}^{\prime}(s)\right) d s-g\left(u_{0}(1)\right) \phi^{\prime}(t), \quad t \in[0,1], \\
& v_{0}(t) \geq \int_{0}^{1} G(t, s) f\left(s, v_{0}(s), u_{0}^{\prime}(s)\right) d s-g\left(v_{0}(1)\right) \phi(t), \quad t \in[0,1], \\
& v_{0}^{\prime}(t) \geq \int_{0}^{1} G_{t}(t, s) f\left(s, v_{0}(s), u_{0}^{\prime}(s)\right) d s-g\left(v_{0}(1)\right) \phi^{\prime}(t), \quad t \in[0,1],
\end{aligned}
$$

where $h(t)=t^{2}, t \in[0,1]$ and $G(t, s)$ is given as in $(3.1)$; 
(2) problem (1.1) has a unique monotone positive solution $u^{*}$ in $P_{h}$;

(3) for any $x_{0}, y_{0} \in P_{h}$, constructing successively the sequences

$$
\begin{aligned}
& x_{n}(t)=\int_{0}^{1} G(t, s) f\left(s, x_{n-1}(s), y_{n-1}^{\prime}(s)\right) d s-g\left(x_{n-1}(1)\right) \phi(t), \quad n=1,2, \ldots \\
& y_{n}(t)=\int_{0}^{1} G(t, s) f\left(s, y_{n-1}(s), x_{n-1}^{\prime}(s)\right) d s-g\left(y_{n-1}(1)\right) \phi^{\prime}(t), \quad n=1,2, \ldots
\end{aligned}
$$

we have $\left\|x_{n}-u^{*}\right\| \rightarrow 0$ and $\left\|y_{n}-u^{*}\right\| \rightarrow 0$ as $n \rightarrow \infty$.

Sketch of the proof Consider two operators $A, B$ defined in the proof of Theorem 3.2. Similarly, from $\left(\mathrm{H}_{1}\right),\left(\mathrm{H}_{2}\right)$, we obtain that $A: P \times P \rightarrow P$ is a mixed monotone operator and $B: P \rightarrow P$ is increasing. From $\left(\mathrm{H}_{5}\right)$, we have

$$
A\left(\lambda u, \lambda^{-1} v\right) \dot{\geq} \lambda A(u, v) ; \quad B(\lambda u) \dot{\geq} \lambda^{\alpha} B u, \quad \text { for } \lambda \in(0,1), u, v \in P .
$$

Since $f(t, 0,2) \not \equiv 0$, we get $\int_{0}^{1} s^{2} f(s, 0,2) d s>0$, and in sequence, $c_{2} \geq c_{1}>0$, here $c_{1}, c_{2}$ are defined in the proof of Theorem 3.2. So we can easily prove that $A(h, h) \in P_{h}$. From $\left(\mathrm{H}_{6}\right)$, we know that $-g(1)>0$, and from the proof of Theorem 3.2 we get $B h \in P_{h}$. For $u, v \in P$ and any $t \in[0,1]$, from $\left(\mathrm{H}_{6}\right)$,

$$
\begin{aligned}
A(u, v)(t)= & \int_{0}^{1} G(t, s) f\left(s, u(s), v^{\prime}(s)\right) d s \\
& \leq \int_{0}^{1} \frac{1}{2} t^{2} s f\left(s, u(s), v^{\prime}(s)\right) d s \leq \frac{1}{2} t^{2} \int_{0}^{1} s \sigma d s \\
& =\frac{1}{4} \sigma t^{2} \leq \frac{1}{4} t^{2}[-g(u(1))]=\frac{3}{4}[-g(u(1))] \frac{1}{3} t^{2} \\
\leq & \frac{3}{4}[-g(u(1))] \phi(t)=\frac{3}{4} B u(t), \\
(A(u, v))^{\prime}(t) & =\int_{0}^{1} G_{t}(t, s) f\left(s, u(s), v^{\prime}(s)\right) d s \\
& \leq \int_{0}^{1} t s f\left(s, u(s), v^{\prime}(s)\right) d s \leq t \int_{0}^{1} s \sigma d s \\
& =\frac{1}{2} \sigma t \leq \frac{1}{2} t[-g(u(1))] \leq[-g(u(1))] \phi^{\prime}(t)=(B u)^{\prime}(t) .
\end{aligned}
$$

Let $\delta_{0}=1$. Then

$$
A(u, v)(t) \leq \delta_{0} B u(t), \quad(A(u, v))^{\prime}(t) \leq \delta_{0}(B u)^{\prime}(t), \quad t \in[0,1] .
$$

Therefore, we get $A(u, v) \leq \delta_{0} B u$ for $u, v \in P$. Finally, an application of Lemma 2.5 implies: there exist $u_{0}, v_{0} \in P_{h}$ and $r \in(0,1)$ such that $r v_{0} \dot{\leq} u_{0} \dot{<} v_{0}, u_{0} \dot{\leq} A\left(u_{0}, v_{0}\right)+B u_{0} \dot{\leq} A\left(v_{0}, u_{0}\right)+$ $B v_{0} \dot{\leq} v_{0}$; the operator equation $A(u, u)+B u=u$ has a unique solution $u^{*}$ in $P_{h}$; for any initial values $x_{0}, y_{0} \in P_{h}$, constructing successively the sequences

$$
x_{n}=A\left(x_{n-1}, y_{n-1}\right)+B x_{n-1}, \quad y_{n}=A\left(y_{n-1}, x_{n-1}\right)+B y_{n-1}, \quad n=1,2, \ldots,
$$


we have $x_{n} \rightarrow u^{*}$ and $y_{n} \rightarrow u^{*}$ as $n \rightarrow \infty$. That is,

$$
\begin{aligned}
& u_{0}(t) \leq \int_{0}^{1} G(t, s) f\left(s, u_{0}(s), v_{0}^{\prime}(s)\right) d s-g\left(u_{0}(1)\right) \phi(t), \quad t \in[0,1], \\
& u_{0}^{\prime}(t) \leq \int_{0}^{1} G_{t}(t, s) f\left(s, u_{0}(s), v_{0}^{\prime}(s)\right) d s-g\left(u_{0}(1)\right) \phi^{\prime}(t), \quad t \in[0,1], \\
& v_{0}(t) \geq \int_{0}^{1} G(t, s) f\left(s, v_{0}(s), u_{0}^{\prime}(s)\right) d s-g\left(v_{0}(1)\right) \phi(t), \quad t \in[0,1], \\
& v_{0}^{\prime}(t) \geq \int_{0}^{1} G_{t}(t, s) f\left(s, v_{0}(s), u_{0}^{\prime}(s)\right) d s-g\left(v_{0}(1)\right) \phi^{\prime}(t), \quad t \in[0,1]
\end{aligned}
$$

problem (1.1) has a unique positive solution $u^{*}$ in $P_{h}$ and $u^{*}(t)$ is monotone increasing; for any $x_{0}, y_{0} \in P_{h}$, constructing successively the sequences

$$
\begin{aligned}
& x_{n}(t)=\int_{0}^{1} G(t, s) f\left(s, x_{n-1}(s), y_{n-1}^{\prime}(s)\right) d s-g\left(x_{n-1}(1)\right) \phi(t), \quad n=1,2, \ldots, \\
& y_{n}(t)=\int_{0}^{1} G(t, s) f\left(s, y_{n-1}(s), x_{n-1}^{\prime}(s)\right) d s-g\left(y_{n-1}(1)\right) \phi^{\prime}(t), \quad n=1,2, \ldots
\end{aligned}
$$

we have $\left\|x_{n}-u^{*}\right\| \rightarrow 0$ and $\left\|y_{n}-u^{*}\right\| \rightarrow 0$ as $n \rightarrow \infty$.

Remark 3.4 Comparing Theorems 3.2-3.3 with the main results in [17], we provide some alternative approaches to study the same type of problems under different conditions. Our results can guarantee the existence of a unique monotone positive solution and the existence of upper-lower solutions, which are seldom seen in the literature.

\section{Examples}

To illustrate how our main results can be used in practice we present two examples.

Example 4.1 Consider the following fourth-order boundary value problem:

$$
\left\{\begin{array}{l}
u^{(4)}(t)=u^{\frac{1}{2}}(t)+\left[u^{\prime}(t)+2\right]^{-\frac{1}{3}}+2, \quad 0<t<1, \\
u(0)=u^{\prime}(0)=0 \\
u^{\prime \prime}(1)=0, \quad u^{(3)}(1)=-\frac{u(1)}{1+u(1)}-1 .
\end{array}\right.
$$

Obviously, problem (4.1) fits the framework of problem (1.1). In this example, let

$$
f(t, x, y)=x^{\frac{1}{2}}+(y+1)^{-\frac{1}{3}}+2, \quad g(x)=-\frac{x}{1+x}-1, \quad \alpha=\frac{1}{2}, \quad \sigma=2 .
$$

Obviously, $f:[0,1] \times[0,+\infty) \times[0,+\infty) \rightarrow[0,+\infty)$ is continuous and $g:[0,+\infty) \rightarrow$ $(-\infty, 0]$ is continuous. And $f(t, x, y)$ is increasing in $x \in[0,+\infty)$ for fixed $t \in[0,1]$ and $y \in[0,+\infty)$, decreasing in $y \in[0,+\infty)$ for fixed $t \in[0,1]$ and $x \in[0,+\infty)$, and $g(x)$ is decreasing in $x \in[0,+\infty)$. Besides, for $\lambda \in(0,1), t \in[0,1], x, y \in[0,+\infty)$, we have

$$
\begin{aligned}
& g(\lambda x)=-\frac{\lambda x}{1+\lambda x}-1 \leq-\frac{\lambda x}{1+x}-\lambda=\lambda g(x), \\
& f\left(t, \lambda x, \lambda^{-1} y\right)=\lambda^{\frac{1}{2}} x^{\frac{1}{2}}+\lambda^{\frac{1}{3}}(y+\lambda)^{-\frac{1}{3}}+2 \geq \lambda^{\frac{1}{2}}\left[x^{\frac{1}{2}}+(y+1)^{-\frac{1}{3}}+2\right]=\lambda^{\alpha} f(t, x, y) .
\end{aligned}
$$


Moreover,

$$
f(t, x, y)=x^{\frac{1}{2}}+(y+1)^{-\frac{1}{3}}+2 \geq \sigma=2 \geq \frac{x}{1+x}+1=-g(x)>0 .
$$

Hence all the conditions of Theorem 3.2 are satisfied. An application of Theorem $3.2 \mathrm{im}$ plies that problem (4.1) has a unique monotone positive solution in $P_{h}$, where $P_{h}$ is the same set in Section 3 and $h(t)=t^{2}, t \in[0,1]$.

Example 4.2 Consider the following fourth-order boundary value problem:

$$
\left\{\begin{array}{l}
u^{(4)}(t)=\cos ^{2} t+\frac{u(t)}{1+u(t)}+\frac{1}{2+u^{\prime}(t)}, \quad 0<t<1, \\
u(0)=u^{\prime}(0)=0, \\
u^{\prime \prime}(1)=0, \quad u^{(3)}(1)=-[u(1)]^{\frac{1}{3}}-3 .
\end{array}\right.
$$

Obviously, problem (4.2) fits the framework of problem (1.1). In this example, let

$$
f(t, x, y)=\cos ^{2} t+\frac{x}{1+x}+\frac{1}{2+y}, \quad g(x)=-x^{\frac{1}{3}}-3, \quad \alpha=\frac{1}{3}, \quad \sigma=3 .
$$

Obviously, $f:[0,1] \times[0,+\infty) \times[0,+\infty) \rightarrow[0,+\infty)$ is continuous and $g:[0,+\infty) \rightarrow$ $(-\infty, 0]$ is continuous. And $f(t, x, y)$ is increasing in $x \in[0,+\infty)$ for fixed $t \in[0,1]$ and $y \in[0,+\infty)$, decreasing in $y \in[0,+\infty)$ for fixed $t \in[0,1]$ and $x \in[0,+\infty)$, and $g(x)$ is decreasing in $x \in[0,+\infty)$. Besides, for $\lambda \in(0,1), t \in[0,1], x, y \in[0,+\infty)$, we have

$$
\begin{aligned}
& g(\lambda x)=-\lambda^{\frac{1}{3}} x^{\frac{1}{3}}-3 \leq \lambda^{\frac{1}{3}}\left(-x^{\frac{1}{3}}-3\right)=\lambda^{\alpha} g(x), \\
& f\left(t, \lambda x, \lambda^{-1} y\right)=\cos ^{2} t+\frac{\lambda x}{1+\lambda x}+\frac{1}{2+\lambda^{-1} y} \geq \cos ^{2} t+\frac{\lambda x}{1+x}+\frac{\lambda}{2+y} \geq \lambda f(t, x, y) .
\end{aligned}
$$

Moreover,

$$
f(t, x, y)=\cos ^{2} t+\frac{x}{1+x}+\frac{1}{2+y} \leq \sigma=3 \leq x^{\frac{1}{3}}+3=-g(x) .
$$

Hence all the conditions of Theorem 3.3 are satisfied. An application of Theorem $3.3 \mathrm{im}$ plies that problem (4.2) has a unique monotone positive solution in $P_{h}$, where $P_{h}$ is the same set in Section 3 and $h(t)=t^{2}, t \in[0,1]$.

Remark 4.3 Examples 4.1, 4.2 imply that there are many functions that satisfy the conditions of Theorems 3.2, 3.3. So the conditions of our results are easy to check.

\section{Competing interests}

The authors declare that they have no competing interests.

\section{Authors' contributions}

The authors declare that the study was realized in collaboration with the same responsibility. All authors read and approved the final manuscript.

\section{Author details}

'School of Mathematical Sciences, Shanxi University, Taiyuan, Shanxi 030006, P.R. China. ${ }^{2}$ School of Computer and Information Technology, Shanxi University, Taiyuan, Shanxi 030006, P.R. China. 


\section{Acknowledgements}

This work was completed when the first author visited College of William and Mary in 2015, and he would like to thank CWM for warm hospitality, and are greatly indebted to Prof. Junping Shi for many helpful suggestions. The first author was partially supported by International Science and Technology Cooperation Projects of Shanxi (2015081020). The second author was partially supported by the Youth Science Foundation of China (11201272) and Shanxi Province Science Foundation (2015011005).

\section{Received: 30 March 2015 Accepted: 29 May 2015 Published online: 19 June 2015}

\section{References}

1. Agarwal, RP: On fourth-order boundary value problems arising in beam analysis. Differ. Integral Equ. 2, 91-110 (1989)

2. Agarwal, RP, Chow, YM: Iterative methods for a fourth order boundary value problem. J. Comput. Appl. Math. 10, 203-217 (1984)

3. Alves, E, Ma, TF, Pelicer, ML: Monotone positive solutions for a fourth order equation with nonlinear boundary conditions. Nonlinear Anal. 71, 3834-3841 (2009)

4. Bai, Z: The upper and lower solution method for some fourth-order boundary value problems. Nonlinear Anal. 67, 1704-1709 (2007)

5. Graef, JR, Yang, B: Positive solutions of a nonlinear fourth order boundary value problem. Commun. Appl. Nonlinear Anal. 14(1), 61-73 (2007)

6. Li, Y: Two-parameter nonresonance condition for the existence of fourth-order boundary value problems. J. Math Anal. Appl. 308, 121-128 (2005)

7. Liu, B: Positive solutions of fourth-order two-point boundary value problems. Appl. Math. Comput. 148, 407-420 (2004)

8. Ma, R, Wang, H: On the existence of positive solutions of fourth-order ordinary differential equations. Appl. Anal. 59, 225-231 (1995)

9. Pei, M, Chang, SK: Monotone iterative technique and symmetric positive solutions for a fourth-order boundary value problem. Math. Comput. Model. 51, 1260-1267 (2010)

10. Yang, B: Positive solutions for the beam equation under certain boundary conditions. Electron. J. Differ. Equ. 2005, 78 (2005)

11. Yao, Q: Positive solutions for eigenvalue problems of fourth-order elastic beam equations. Appl. Math. Lett. 17, 237-243 (2004)

12. Zhang, XP: Existence and iteration of monotone positive solutions for an elastic beam with a corner. Nonlinear Anal. 10, 2097-2103 (2009)

13. Amster, P, Cárdenas Alzate, PP: A shooting method for a nonlinear beam equation. Nonlinear Anal. 68, 2072-2078 (2008)

14. Jankowski, T, Jankowski, R: Multiple solutions of boundary-value problems for fourth-order differential equations with deviating arguments. J. Optim. Theory Appl. 146, 105-115 (2010)

15. Yang, L, Chen, $\mathrm{H}$, Yang, X: The multiplicity of solutions for fourth-order equations generated from a boundary condition. Appl. Math. Lett. 24, 1599-1603 (2011)

16. Zhai, CB, Song, RP, Han, QQ: The existence and the uniqueness of symmetric positive solutions for a fourth-order boundary value problem. Comput. Math. Appl. 62, 2639-2647 (2011)

17. Li, S, Zhang, X: Existence and uniqueness of monotone positive solutions for an elastic beam equation with nonlinear boundary conditions. Comput. Math. Appl. 63, 1355-1360 (2012)

18. Cabada, A, Tersian, S: Multiplicity of solutions of a two point boundary value problem for a fourth-order equation Appl. Math. Comput. 219, 5261-5267 (2013)

19. Wang, WX, Zheng, YP, Yang, H, Wang, JX: Positive solutions for elastic beam equations with nonlinear boundary conditions and a parameter. Bound. Value Probl. 2014, Article ID 80 (2014)

20. Liu, RK, Ma, RY: Existence of positive solutions for an elastic beam equation with nonlinear boundary conditions J. Appl. Math. 2014, Article ID 972135 (2014)

21. Zhai, CB, Hao, MR: Fixed point theorems for mixed monotone operators with perturbation and applications to fractional differential equation boundary value problems. Nonlinear Anal. 75, 2542-2551 (2012)

22. Guo, D, Lakshmikantham, V: Nonlinear Problems in Abstract Cones. Academic Press, New York (1988)

23. Guo, D, Lakskmikantham, V: Coupled fixed points of nonlinear operators with applications. Nonlinear Anal. 11(5), 623-632 (1987) 